\title{
Médiévales
}

Langues, Textes, Histoire

66 | printemps 2014

Harmonie Disharmonie

\section{Esthétique de l'entremêlement dans les portraits du jeune Lancelot et de Claudas de la Terre Déserte dans le Lancelot en prose}

The Aesthetic of "Entanglement » in the Portraits of the Young Lancelot and of Claudas de la Terre Déserte in the Prose Lancelot

\section{Ludivine Jaquiery}

\section{OpenEdition}

\section{Journals}

\section{Electronic version}

URL: https://journals.openedition.org/medievales/7228

DOI: $10.4000 /$ medievales.7228

ISSN: $1777-5892$

Publisher

Presses universitaires de Vincennes

\section{Printed version}

Date of publication: 30 June 2014

Number of pages: 91-104

ISBN: 978-2-84292-405-8

ISSN: 0751-2708

\section{Electronic reference}

Ludivine Jaquiery, "Esthétique de l'entremêlement dans les portraits du jeune Lancelot et de Claudas de la Terre Déserte dans le Lancelot en prose", Médiévales [Online], 66 | printemps 2014, Online since 05 July 2016, connection on 23 April 2022. URL: http://journals.openedition.org/medievales/7228; DOI: https://doi.org/10.4000/medievales.7228 


\title{
Esthétique de l'entremêlement dans les portraits du jeune Lancelot et de Claudas de la Terre Déserte dans le Lancelot en prose
}

\begin{abstract}
Il imagine un corps comme un manteau. On s'en débarrasserait, on pourrait le pendre, l'abandonner à une patère lorsqu'il ne convient plus. En choisir un autre, qui vous va mieux, qui révèle plus précisément, plus élégamment la silhouette de votre âme. La taille de votre cœur.

G. Delacourt, La Première Chose qu'on regarde.
\end{abstract}

Les portraits dans le Lancelot en prose, œuvre monumentale du $\mathrm{XIII}^{\mathrm{e}}$ siècle, sont suffisamment rares pour que ceux de Claudas de la Terre Déserte et de Lancelot enfant, au début de la Marche de Gaule, interpellent le lecteur ${ }^{1}$. Leur proximité dans le texte invite tout d'abord à les analyser en regard l'un de l'autre, bien que Claudas soit l'ennemi de Lancelot et du royaume arthurien, tandis que le second est amené à incarner le modèle du chevalier courtois. Les portraits sont construits sur un effet de disharmonie qu'il conviendrait aux deux personnages de dépasser. Si cette entreprise est un échec pour Claudas, Lancelot parvient à maitriser ses excès, à les intégrer, sans les effacer pour autant.

Au tout début de la Marche de Gaule, nous apprenons dans un récit rétrospectif que Claudas, roi de Bourges, au côté d'Aramont, roi de Gaule, a été défait par Uterpendragon, le père d'Arthur, allié à Hoël de Petite Bretagne. Les terres de Claudas ont été ravagées par Uterpendragon, d'où l'épithète «de la Terre Déserte». Dans le présent de la narration, Claudas s'est reconstitué une armée et entend bien laver l'affront qui lui a été fait. Les objets de sa vengeance sont deux frères, des vassaux du roi Arthur, Ban

1. Dans le cycle du Lancelot-Graal, la Marche de Gaule est le début du Lancelot propre, qui suit le Joseph d'Arimathie (Histoire du Saint Graal), le Merlin et les Premiers Faits du roi Arthur (Suite du Merlin). Le Lancelot est suivi de la Quête du Saint Graal et de la Mort du roi Arthur. Le portrait de Claudas occupe les p. 54-56 du t. VII du Lancelot, roman en prose du XII $e^{e}$ siècle, éd. A. Мicha, Genève, 1980, celui de Lancelot figure aux p. 70-75. Nous tenons à remercier la Professeure Yasmina Foehr-Janssens et le Professeur Christopher Lucken pour leurs conseils éclairés. 
de Benoïc et Bohort de Gaunes, respectivement père de Lancelot, et père de Lionel et Bohort.

En promettant des terres au sénéchal de Ban s'il accepte de trahir son seigneur, Claudas s'empare de la cité de Bénoïc et la brûle, sous le regard désespéré de Ban qui part en quête d'aide avec sa femme et Lancelot qui n'est encore qu'un nourrisson. Au spectacle de la ville en flammes, le roi meurt de chagrin. C'est à ce moment que Lancelot est enlevé sous les yeux de sa mère impuissante par la Dame du Lac qui s'occupera de lui et se chargera de son éducation. Claudas anéantit Bohort de Gaunes. Les deux enfants de ce dernier - Lionel et Bohort - sont sauvés, mais Claudas les enfermera dans une tour pour les empêcher de lui nuire. Claudas est l'ennemi du lignage de Lancelot: il a dépouillé Lancelot et ses cousins de leur héritage.

Le portrait que le narrateur dresse de Claudas est tripartite. Les qualités et défauts de l'usurpateur encadrent la partie centrale, dévolue à sa description physique et marquée par un fort contraste: son visage est laid, tandis que le reste de son corps est parfait. Claudas est un être double, et l'ensemble de la description s'attache à rendre son ambivalence. Cela est remarquable dès la première mention du personnage, qualifié comme «moult boins chevaliers et moult sages, mais moult estoit traïtres ${ }^{2} »$. Il serait un modèle chevaleresque, si la traîtrise ne neutralisait pas les deux premiers qualificatifs.

Le portrait apparaît à un moment charnière du récit. En effet, comme nous venons de le rappeler, Claudas incarne l'ennemi par excellence. Pourtant, après cette description, Claudas de la Terre Déserte part incognito à la cour d'Arthur pour évaluer les forces de ce dernier et constater par lui-même si sa renommée est méritée. Fasciné par les qualités d'Arthur, il envisage de prendre ses valeurs pour modèle. Il sera même prêt à rendre leurs terres à Lionel et Bohort mais un quiproquo, dû à l'intervention de la Dame du Lac qui mène à la mort de Dorin, le fils de Claudas, le ramènera à ses premières dispositions

Le roi de la Terre Déserte disparaît par la suite de la narration, s'effaçant devant les exploits de Lancelot et ses amours avec Guenièvre. Quand il reparaît sur la scène des aventures, c'est pour insulter la reine Guenièvre car il est au courant de sa relation avec Lancelot. Ce dernier choisit de venger la reine, arguant qu'il souhaite récupérer les terres de son père et son oncle dont Claudas l'a dépouillé, ainsi que ses cousins. Arthur le soutient et décide de se rendre en Gaule et d'entrer en guerre. Claudas, vaincu, est contraint de fuir à Rome où il s'enfonce définitivement dans l'oubli. Si le traitement du personnage de Claudas confère à ce dernier la potentialité d'agir conformément au bien, une telle perspective n'est jamais

2. Lancelot, t. VII, p. 2. actualisée; la fonction de Claudas dans la narration ne le permet pas. Le rôle de celui-ci s'achève avec sa défaite: présenté tout d'abord comme l'ennemi de Lancelot, Claudas devient l'ennemi d'Arthur, ce qui le mène à la ruine, conformément à un schéma narratif qui remonte à l'Historia regum Britanniae de Geoffroy de Monmouth.

Lorsque Claudas revient de son expédition secrète chez Arthur, le récit le quitte pour s'intéresser à Lancelot, désormais sous la garde de la Dame du Lac. Il est question de son éducation et c'est là que son portrait est dressé.

Le portrait de Lancelot insiste surtout sur son apparence. La description physique occupe trois pages dans l'édition de Micha, contre une dizaine de lignes pour Claudas. Relevons deux éléments relatifs à son éducation: il apprend à chasser et maîtrise le jeu d'échecs, en bon fils de roi. Ces deux occupations sont également mentionnées dans le portrait de Claudas, comme nous le préciserons plus loin. Le portrait se clôt sur «les teches du cuer ${ }^{3} »$. Mais le texte ne s'étend pas sur ce sujet. Largesse, douceur et débonnaireté, actions guidées par le bien, telles sont les valeurs du jeune Lancelot. Il n'est guère besoin d'en dire plus : la «samblance» de Lancelot parle pour lui. Le narrateur décrit ensuite le teint du jeune Lancelot, sa bouche, ses dents, son menton, son nez, ses yeux, son front, ses sourcils, ses cheveux, son cou, ses épaules, sa poitrine, ses bras, ses mains, ses hanches, ses cuisses, ses jambes et enfin ses pieds. Le maître-mot de la description est la «mesure»: la bouche est «petite par mesure», le nez «par mesure lonc», les cheveux «crespés et cleirs par mesure», le cou «a la mesure» des épaules et du corps; pour ses bras, «de char i ot a mesure ${ }^{4}$.

Le portrait de Lancelot est effectué selon un mouvement descendant. Il répond ainsi aux modèles préconisés par les arts poétiques médiévaux. Aussi, avant d'examiner les liens qui rapprochent ce portrait de celui de Claudas, malgré leur asymétrie, il convient de rappeler brièvement les préceptes qui gouvernent l'art du portrait littéraire au XIII ${ }^{\mathrm{e}}$ siècle.

\section{La description des personnages d'après les arts poétiques}

La réflexion des théoriciens médiévaux en matière de poétique est nourrie principalement par le De inventione de Cicéron, la Rhétorique à Hérennius du Pseudo-Cicéron et l'Art poétique d'Horace. Nous allons nous appuyer sur la Poetria nova de Geoffroy de Vinsauf et sur l'Ars versificatoria

3. Lancelot, t. VII, p. 74

4. Lancelot, t. VII, p. 72-73. 
de Matthieu de Vendôme ${ }^{5}$. Sur le plan du contenu, le portrait d'un personnage peut se concentrer sur l'aspect physique, selon un mouvement descendant allant de la tête aux pieds, et sur les qualités morales ${ }^{6}$. Les deux ne sont pas nécessairement présents, d'autant que le corps tend à traduire visuellement les dispositions intérieures.

La description vise à faire l'éloge ou à blâmer une personne, notamment dans le but d'émouvoir. Matthieu de Vendôme, dans son Ars versificatoria, s'en souvient ${ }^{7}$. Pour lui, la description de personne n'est jamais gratuite ${ }^{8}$ : elle n'est pas qu'un simple ornement visant à l'amplificatio de la narration ${ }^{9}$. Dans sa Poetria nova, Geoffroy de Vinsauf se contente de donner des exemples de description, le sujet étant alors rebattu ${ }^{10}$. Mais l'habileté de l'auteur réside dans sa capacité à respecter le modèle théorique tout en le renouvelant: Jean-Yves Tilliette a montré comment, avec un portrait d'Hélène, parangon de beauté, Joseph d'Exeter parvient à intégrer quelque chose d'inattendu, puisque sa beauté «connote sa méchanceté ${ }^{11}$ ». Matthieu de Vendôme juxtapose le portrait d'Hélène et celui de Béroé et choisit de les «traite[r] en manière de pendants antithétiques [...]», comme «d'autres se sont plu à des contrastes analogues: ainsi, dans le Jeu de la Feuillée, Adam le Bossu oppose en deux descriptions la beauté de Maroie en sa jeunesse et le dépérissement de ses attraits à l'approche de l'âge ${ }^{12}{ }^{\prime}$. Et Douglas Kelly a bien montré l'ingéniosité de Chrétien de Troyes, qui joue sur le stéréotype de la description en y mêlant des «anomalies »: pour ne citer qu'un exemple, Énide porte des vêtements usés et inadéquats à sa

5. Les chapitres sur la description de personnages occupent les p. 118-143 (MatthiEU de Vendôme, Ars versificatoria, I. 38-I. 92), les p. 214-217 (Geoffroy de Vinsauf, Poetria nova, v. 554-667) et les p. 75-82 (introduction) des Arts poétiques $d u$ XII ${ }^{e}$ et $d u$ XIII ${ }^{e}$ siècles, recherches et documents sur la technique littéraire du Moyen Âge, éd. E. FARA Paris, 1924; Matthew of Vendôme Ars versificatoria (The Art of the Verse, E. E. Far. Paris, 924; Mathew of Vents sur in technique littercire du Mors Milwaukee, 1981, p. 27-51; Poetria Nova of Geoffrey of Vinsauf, trad. M. F. Nims, Toronto, 1967, p. 36-39

6. Cf. Matthieu de Vendôme, Ars Versificatoria, I. 74, et Geoffroy de Vinsauf, Poetria nova, v. 598-599. Notons que les descriptions diffèrent suivant les caractéristiques des personnes: par exemple, un portrait de jeunesse sera différent d'un portrait de vieillesse (MatThieu de Vendôme, Ars versificatoria, I. 41, qui se fonde sur l'Art poétique d'Horace, v. 114 sq.).

7. MatTHifu de Vendôme, Ars versificatoria, I. 59.

8. Ibid., I. 38.

9. La description est un procédé d'amplification. Jean-Yves Tilliette (Des mots à la Parole : une lecture de la Poetria nova de Geoffroy de Vinsauf, Genève, 2000, p. 90) souligne que l'amplification ne vise pas seulement à rallonger le récit, elle met en lumière le mieux possible ce qui est décrit.

10. Geoffroy de Vinsauf, Poetria nova, v. 622-623: la description est «res quasi trita et vetus», «triviale et ancienne».

11. J.-Y. Tilliette, Des mots à la Parole..., p. 94; cf. ID., «La descriptio Helenae dans la poésie latine du XII ${ }^{\mathrm{e}}$ siècle», Bien dire et bien aprandre, 11 (1993), p. 419-432.

12. E. FARAL, Les Arts poétiques du XII et du XIII' siècles..., p. 77. beauté, mais les trous de sa parure dévoilent habilement le corps superbe de la jeune femme ${ }^{13}$. Il nous semble que le rédacteur du Lancelot en prose joue lui aussi sur les possibilités d'un usage contrasté des stéréotypes offertes par l'exercice rhétorique du portrait. Lancelot et Claudas sont présentés de telle manière que leurs portraits suffisent à eux seuls à mettre en place les enjeux du récit en ce qui les concerne.

\section{Démesure et entremêlement}

Nous avons vu, dans l'analyse des portraits physiques, que Lancelot est proportionné, harmonieux, contrairement à Claudas, qui est mi-beau, mimonstrueux. Le premier est donc le parfait exemple du héros, beau et bon, tandis que le second est l'image de l'ennemi, dont l'ambivalence se traduit par un corps et une tête en désaccord l'un avec l'autre. Pourtant, Lancelot a lui aussi un défaut physique, une poitrine un peu trop large. Si ce défaut est justifié par la reine Guenièvre, il l'est également par Claudas, dans un dialogue mêlé à son portrait. À cela près que le discours de Claudas a une portée générale et ne vise pas Lancelot en particulier. C'est un des éléments qui nous autorise à associer les deux descriptions. L'adjectif «entremellé», qui fait retour, en est un second.

\section{Le «manteau» du cœur: la poitrine de Lancelot}

Nous avons insisté sur la perfection de Lancelot et la «mesure» qui caractérise ses proportions. Pourtant, un élément détonne, soit la largeur démesurée de sa poitrine, qualifiée comme un défaut et signalée comme tel par ses contemporains ${ }^{14}$ :

[...] et le pis teil que en nul cors ne trovast on ne si large ne si gros ne si espés; ne en lui ne trovast ja nus hom plus que reprendre, ains disoient tout chil qui le veioient que, s'il fust un poi mains garnis de pis, plus en fust ratalentables et plaisans.

Comme pour mieux souligner cette imperfection, mais tout en la justifiant, le narrateur aménage au sein de ce portrait une curieuse ellipse temporelle en donnant la parole à Guenièvre qui, à ce moment du récit, ne connaît

13. D. KelLy, «La conjointure de l'anomalie et du stéréotype: un modèle de l'invention dans les romans arthuriens en vers», Cahiers de Recherches Médiévales et Humanistes, 14 (2007), p. 25-39; ID., «The Art of Description», dans N. J. LACY, D. Kelly, K. BusBY éd., The Legacy of Chrétien de Troyes, Amsterdam, 1988, vol. I, ch. 8, p. 191-221.

14. Lancelot, t. VII, p. 73. 
pas encore Lancelot. On apprend que Guenièvre, lorsqu'elle sera l'amie de Lancelot, légitimera cette disproportion ${ }^{15}$ :

[...] Diex ne li avoit pas donné pis a outrage de grant ne de gros ne d'espesse qui i fust, car autresi estoit grans li cuers a son endroit, si covenist que il crevast par estovoir, se il n'eust teil estage ou il se reposast a se mesure [...].

Le cœur et le corps sont à la mesure l'un de l'autre. Étant donné l'importance de son amour et tout ce que celui-ci l'incite à accomplir, il fallait nécessairement que son corps puisse loger ce cœur extraordinaire. L'imperfection physique a sa cause dans une analogie entre le corps et le cœur, marqués par un semblable excès.

Cette précision est d'autant plus intéressante qu'elle fait écho à un propos tenu par Claudas, justement. En réponse à une question de ses proches sur les raisons de son célibat, Claudas affirme qu'il ne se marie pas car il désire vivre vieux. Selon lui, son corps n'est pas en mesure de supporter ce qu'un cœur amoureux doit entreprendre: il s'épuiserait. Voilà pourquoi, lui qui a été amoureux dans le passé, a finalement abandonné l'amour. Claudas précise que, si on pouvait posséder un corps assez vigoureux pour endurer les volontés d'un cœur amoureux, il se mettrait aussitôt au service d'amour et serait le meilleur chevalier du monde ${ }^{16}$.

$[\ldots]$ «mais se la forche del cors fust si grans que ele peust acomplir les hardemens del cuer, je amasse par amours toute ma vie et passase tous les preudommes de toutes iceles proeches qui peuent estre en cors de boin chevalier: car il ne puet estre tres preus d'armes, s'il n'aime tres loiaument. Et je connois tant mon cuer que je amasse loiaument sour tous loiaus hommes qui par amours amassent.»

Lancelot réalise en revanche dans son corps le programme jugé impossible par son ennemi juré. Il devient en quelque sorte le Claudas exemplaire imaginé par ce dernier.

Le caractère exceptionnel de Lancelot, qui le porte physiquement sur lui, est ici souligné. En effet, c'est son amour pour Guenièvre et les exploits qu'il accomplit en son nom qui font la particularité du chevalier ${ }^{17}$.

15. Lancelot, t. VII, p. 73

16. Lancelot, t. VII, p. 55

17. L'idée est reprise un peu plus loin, dans le portrait de Lancelot, mais avec la «joie» comme moteur: «[...] quant il estoit en sa grant joie, $[\ldots]$ riens nule ses cuers n'oseroit emprendre que ses cors ne peust mener a fin [...]» (Lancelot, t. VII, p. 74). Galehaut justifiera quant à lui sa grande taille par le caractère exceptionnel de son cœur: «J'ai esté li plus merveillos hom qui onques fust et si ai eu si merveillos cuer que, s'il fust en un petit cors, je ne voi pas coment il peust durer, kar onques de nule grant emprise ne le trovai lache ne pereçeus,
Mais cette poitrine de taille excessive, à première vue disharmonieuse, est également le signe d'une sorte d'hybris caractéristique du héros. Tout excès est potentiellement dangereux. Cependant, Lancelot l'intègre et en fait son signe caractéristique, ce qui le distingue positivement des autres héros. Le rapport à son propre corps que construit de son côté Claudas, laisse penser, au contraire, que le cœur s'épuise faute d'un corps à sa mesure. Or la description dont il est l'objet signale que son corps est parfait; point de disproportion dans la taille de sa poitrine ${ }^{18}$ :

[...] mais les espaules, le pis et tout l'autre cors ot il si bel et si bien fait com l'en poroit miex deviser en nul homme.

Le traitement contrasté des deux portraits aboutit donc à une sorte de renversement de l'opposition entre mesure et démesure. Pour qu'un chevalier soit le meilleur, l'excès, une fois dompté, est nécessaire, car en matière de cœur, la démesure fait loi. Ce qui est mesuré chez Claudas, son corps, ne fait pas l'objet d'un éloge. En revanche, si la trop large poitrine de Lancelot est valorisée, c'est qu'elle mêle à l'apparence physique un autre aspect, celui du cœur. L'amour, chez Lancelot, est un principe fédérateur qui légitime ce qui, dans d'autres circonstances, aurait été considéré comme disharmonieux. Toutefois, la remarque de Claudas sur l'éclatement possible du cœur laisse aussi entrevoir la possibilité que l'amour pourrait être un facteur de rupture de l'harmonie. Il reste une partie du corps dont il n'a pas encore été question: la tête. Or la description du visage de nos deux héros recèle elle aussi un élément qui permet de réfléchir en termes de «mesure», en lien cette fois avec la notion d'entremêlement.

\section{Deux procédés d'entremêlement: fusion et dualité}

Après une brève recherche des occurrences du verbe «entremêler» - adjectivé ou non - dans les textes français médiévaux ${ }^{19}$, il ressort qu'il est utilisé principalement: dans le domaine esthétique, pour décrire des alliances de couleurs (pour le teint d'un personnage par exemple) ou de motifs; dans le domaine musical, pour traiter du mélange entre chant et musique instrumentale; dans un contexte guerrier, lorsque des armées

mais tos jors enprenant et volonteis assés plus que mes consels ne li osast doner » (Lancelot, éd. A. MıchA, Genève, t. I, 1978, p. 16-17). Son cœur est adapté à son corps, au regard de la prouesse dont il fait preuve, mais ses sentiments ambigus pour Lancelot pourraient également le faire tendre du côté de la figure de l'amoureux. À un amour extraordinaire correspondent un cœur et un corps non moins singuliers, chez Lancelot comme chez Galehaut.

18. Lancelot, t. VII, p. 54.

19. Nous avons consulté pour cela le Corpus de la littérature médiévale des origines au $\mathrm{XV}^{e}$ siècle (Classiques Garnier numérique). 
s'affrontent et qu'il est impossible de distinguer qui est membre de quelle armée; dans l'idée qu'il ne faut pas «entremesler» un lignage; dans le registre amoureux, pour exprimer les symptômes de la maladie d'amour.

Dans ces exemples, deux nuances se dégagent: soit les éléments s'entremêlent de manière à ce que l'on ne distingue plus les différences. C'est le cas dans le contexte guerrier, par exemple, où amis et ennemis se confondent. On peut alors parler d'une harmonisation consistant en une unification des camps opposés. Soit des éléments contraires sont coprésents, tout en s'excluant l'un l'autre, comme par exemple lorsqu'un amant est saisi simultanément de «froidure» et d' «ardure ${ }^{20}$, voire passe d'une sensation à l'autre. La disharmonie règne en maître dans le corps de l'amant.

En ce qui concerne nos deux personnages, ce sont les couleurs du teint de Lancelot et des cheveux de Claudas qui sont entremêlées. Le soin accordé à la «charneure» de Lancelot est manifeste; celle-ci est le fruit d'un mélange parfaitement équilibré de différentes composantes ${ }^{21}$ :

Il fu de moult bel charneure, ne bien blans ne bien bruns, mais entremellés d'un et d'autre: sel puet on apeleir cleirs brunés. Il ot le viaire enluminé de naturel color vermelle, si par mesure que visaument i avoit Diex assise le compaignie de la blanchor et de la brunor et del vermel, que la blancor n'estoit estainte ne enpirie pour la brunor, ne la brunor por la blanchor, ains estoit atenprés l'une de l'autre, et la vermelle color qui par desus estoit enluminoit et soi et les autres colors melees, si que rien n'i avoit trop blanc ne trop brun ne trop vermel, mais igal melleure de .III. ensamble.

La couleur de sa peau est présentée comme le résultat d'un mélange: «ne bien blans, ne bien bruns, mais entremellés d'un et d'autre». Le blanc et le brun, s'équilibrent, ce qui est rendu par l'appellation que l'on peut donner à ce teint, «cleirs brunés ${ }^{22} »$.

20. Cet exemple est tiré du Livre du voir dit de Guillaume de Machaut: «Et si sentoie une froidure / Entremellee d'une ardure / Qui faisoit fremir et suer / Mon corps et ma couleur muer» (éd. et trad. J. ImBS et J. Cerquiglini-Toulet, Paris, 1999, v. 1932-1935). Il s'agit d'un topos, de même que les émotions contraires - joie et tristesse - qui peuvent saisir un amant ou une dame en proie aux tourments de l'amour.

21. Lancelot, t. VII, p. 71-72.

22. Dans l'autre portrait, Lancelot est décrit par le narrateur comme ayant «la chiere clere et brune» (Lancelot, t. I, p. 128-129). Dans l'édition de F. Mosès, une note précise qu'il devrait s'agir ici aussi de «clair-brunet». «La plupart des copistes ont pensé qu'il s'agissait d'épithètes différentes et ont écrit "clair et brun"» (Lancelot du Lac III, La fausse Guenièvre, éd. et trad. F. Mosès et L. Le GuAY, Paris, 1998, p. 251). Si c'est effectivement le cas, est-ce à dire que la volonté d'exprimer, pour le teint, une fusion par le vocabulaire est rare? Sur le plan de l'adjectif même, tous les exemples d'emploi de «brunet» figurant dans le dictionnaire Tobler-Lommatzsch désignent la couleur des cheveux et des sourcils.
L'impression de mesure dans les couleurs qui se mêlent s'accroît encore lorsque, pour qualifier le teint du visage de Lancelot, le «vermeil» s'ajoute aux deux couleurs précédentes. Le blanc, le brun et le rouge sont, «par mesure, en bonne compaignie: si que rien n'i avoit trop blanc ne trop brun ne trop vermel, mais igal melleure de .III. ensamble». Ajoutons à cela que l'alliage de ces couleurs converge vers un éclat idéal. Le rouge «enlumine» le visage. Cette idée est reprise ensuite, «la vermelle color qui par desus estoit enluminoit et soi et les autres colors melees». De même, le brun et le blanc ne «s'éteignent» pas l'un l'autre. La lumière, fondamentale dans l'esthétique médiévale, a ici la part belle ${ }^{23}$. Chaque couleur met donc en valeur les autres sans pour autant qu'il y ait une véritable confusion. Les couleurs sont également présentes, mais elles s'harmonisent.

Comme le souligne Alice Colby, le mélange du vermeil et du blanc est un élément habituel pour les portraits de jeunesse et les portraits féminins ${ }^{24}$. Cette dernière note également la présence du brun, parfois, mais jamais les trois couleurs simultanément. Pour Micheline de Combarieu, la composition de la «charneure» de Lancelot «donne l'idée, non d'une moyenne, mais d'une double participation aux contraires, impliquant même parfois la médiation d'un tierce élément ${ }^{25} »$. Si on suit sa lecture, Lancelot est construit comme une addition de contraires, liée parfois par un troisième élément. Nous pensons que cela révèle pour Lancelot un dépassement des oppositions.

Néanmoins, la perfection de ce teint harmonieux peut subir de brusques et impressionnantes altérations. Lancelot, précise le narrateur, est sujet à la colère. Dans ces cas-là, il passe du côté de l'animalité. Cela présage les grandes scènes de folie, dont l'origine est liée à Guenièvre ${ }^{26}$. Les yeux du garçon semblent être de «carbon espris». On a l'impression que

[...] par mi le pomel des joes li sailloient goutes de sanc toutes vermeilles et fronchoit del neis en sa grant ire autresi com uns chevaus et estregnoit les

23. Sur ce sujet, $c f$. E. De Bruyne, Études d'esthétique médiévale, t. III (Le XIII' siècle), Bruges, 1946

24. A. M. Colвy, The Portrait in Twelfth-Century French Literature, Genève, 1965. Quelques pages sont consacrées au visage et au teint: p. 46-48

25. M. De Combarieu, «Le Lancelot comme roman d'apprentissage: enfance, démesure et chevalerie», dans J. Dufournet éd., Approches du Lancelot en prose, Genève/Paris, 1984, p. 101-136 (p. 129). Elle ajoute à cela les caractéristiques doubles, féminines et masculines, de Lancelot, qu'offre la suite du portrait. Il nous semble que, puisqu'il s'agit d'un portrait d'enfance, à un moment où les différences de genre sont peu marquées, il est peut-être excessif de parler de traits feminins.

26. Nous apprenons que, «quant il se courechoit d'aucune chose que l'en li eust mesfaite, n'estoit lors pas legiere chose de lui apaier» (Lancelot, t. VII, p. 74-75). 
dens ensemble si que il croissoient moult durement, et iert avis que l'alaine qui de sa bouche issoit fust toute vermelle ${ }^{27}[\ldots]$.

Au-delà des grincements de dents et de l'attitude équine, ce qui frappe ici, c'est la dominance de la couleur rouge. Les yeux ressemblent à du charbon enflammé, les joues semblent suinter du sang, l'haleine est vermeille. La belle harmonie des couleurs est rompue lorsque Lancelot perd son calme. Le vermeil prend le dessus sur les autres couleurs et c'est le signe du dérèglement du chevalier. Lancelot ne se souvient pas des violentes ruptures de sa complexion ordinaire; c'est comme s'il était absent à lui-même. Dans la théorie des humeurs, la couleur rouge renvoie également au tempérament sanguin: le sang est prépondérant sur les trois autres humeurs. L'individu de type sanguin possède un caractère impulsif. Victime de «coups de sang », Lancelot subit donc aussi un dérèglement de ses humeurs.

On retrouve une même tendance à l'exagération dans son comportement: il est «li plus dous enfes et li plus deboinaires de tous, la ou deboinairetés se laisoit trover. Mais contre felonnie le trevoit on passeifelon ${ }^{28}{ } \gg$. L'extrême débonnaireté du jeune Lancelot et son intolérance face à la félonie traduisent non seulement son tempérament, la noblesse de son caractère, mais peuvent également être imputables à sa jeunesse: un adulte doit se maîtriser, alors que la jeunesse est la période où les forces excèdent, la sagesse étant moins présente. Philippe de Navarre, dans Les Quatre Âges de l'homme, écrit que, lorsqu'on atteint l'âge moyen, «se doit on amesurer et retraire des folies que l'on a fait en jovant ${ }^{29} »$.

Lorsqu'il est fait état du caractère général de Lancelot, sans l'associer à des circonstances particulières, c'est l'harmonie qui domine. Par exemple, le narrateur se plaît à insister sur le fait que le jeune garçon combine les qualités de l'adulte et de l'enfant ${ }^{30}$ :

[...] si fu tant biax qu'il ne fu nus qui le veist qu'il ne quidast qu'il fust de grignour eage la tierche part que il n'estoit, et avoec che qu'il estoit grans de son eage, si estoit sages et entendans et legiers et outre che que enfes de son eage ne doit estre.

Le topos du puer senex n'est, dans ce contexte, qu'une caractéristique supplémentaire illustrant l'union des contraires chez le futur chevalier. Mais nous insistons une fois encore sur le fait qu'il combine des éléments contraires, qui sont à la fois coprésents et harmonisés.

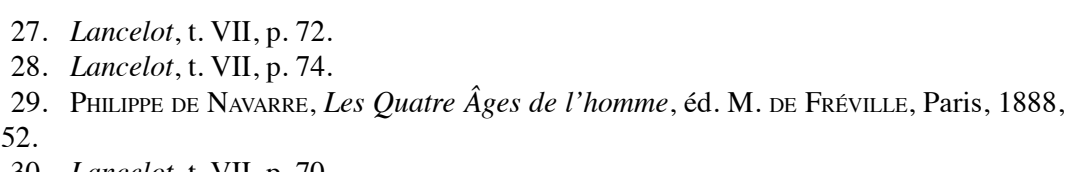

27. Lancelot, t. VII, p. 72

29. PhilipPe de Navarre, Les Quatre Âges de l'homme, éd. M. DE FrévILLE, Paris, 1888 ,

ancelot, t. VII, p. 70
Chez Claudas, nous retrouvons exactement la même construction syntaxique que dans le portrait physique de Lancelot, avec l'adjectif «entremelé», mais cela concerne la couleur de ses cheveux. Ils sont «ne bien noir ne bien rous ${ }^{31}$, mais entremelés d'un et d'autre» (nou soulignons) $)^{32}$. Ils se mélangent sans se fondre. L'effet de l'entremêlement est donc traité différemment. Le brun et le roux paraissent irréconciliables. Aucun développement, comme celui dévolu au teint de Lancelot, ne s'ensuit. L'entremêlement désigne ici une coprésence, sans que les éléments se mêlent véritablement, se mettent en valeur.

Claudas ne participe donc pas d'une union harmonieuse d'éléments divers; au contraire, il n'échappe jamais à un dualisme net ${ }^{33}$.

[...] si avoit le viaire noir et gros et les sorchiex velus et les iex gros et noirs, l'un loig de l'autre. Il avoit le neis court et reskignié et le barbe rousse et les cheveus ne bien noir ne bien rous, mais entremelés d'un et d'autre; si ot le col gros et le bouche grande et les dens clers et enchises; mais les espaules, le pis et tout l'autre cors ot il si bel et si bien fait com l'en poroit miex deviser en nul homme. (Nous soulignons.)

En contraste avec son beau corps, la tête de Claudas - la noirceur de son visage et de ses yeux, leur éloignement l'un de l'autre, ses cheveux roux et noirs, sa bouche grande, ses dents acérées, sa pilosité - évoque l'homme sauvage, la laideur. Claudas semble être le résultat d'un patchwork ${ }^{34}$.

Conformément à ce que nous savons de la relation âme-corps, le portrait moral de Claudas exprime lui aussi son caractère double. Tout d'abord, il est dépeint sous un jour peu flatteur: il est

31. Notons que le roux est une couleur fortement connotée, c'est la couleur de la ruse, du traître. Croiser un personnage roux dans un récit est une incitation à la méfiance. Sur ce sujet, voir notamment les travaux de Michel Pastoureau, et plus exactement son analyse du roux qui symboliserait la combinaison du mauvais jaune et du mauvais rouge, chaque couleur étant ambivalente, ainsi que son étude consacrée à Judas, souvent représenté avec la chevelure rousse: M. PASTOUREAU, «Vers une histoire sociale des couleurs», dans Couleurs, images, Ver (1)

32. Lancelot, t. VII, p. 54

33. Lancelot, t. VII, p. 54.

34. P. MÉNARD, dans une étude consacrée au portrait de la laideur, mentionne d'ailleurs que «si, d'aventures, un de ces figurants aux dépens desquels s'égaient les conteurs n'est pas totalement antipathique, le portrait juxtapose laideur et beauté afin de produire une impressio mélangée. Mais ce sentiment de la complexité humaine reste exceptionnel» (Le Rire et le sourire dans le roman courtois en France au Moyen Âge. 1150-1250), Genève, 1969, p. 542). 
[...] li plus angoissous prinche et li plus avers del monde ne jamés ne dounast, se lors non quant il avoit si grant mestier de gent que consievrer ne s'en pooit ${ }^{35}$.

«Angoissous » et «avers » sont des adjectifs qualificatifs susceptibles d'avoir un sens positif comme négatif, mais le contexte nous enjoint à comprendre le premier terme en mauvaise part, tandis que le second est clairement négatif. La largesse, valeur suprême du seigneur de type arthurien, ne fait pas partie des qualités de Claudas.

La dernière partie de la description qui lui est dévolue est plus avantageuse et ne laisserait pas présager sa félonie, si ce qui précède n'était pas connu du lecteur; c'est un homme de confiance à qui on reconnaît également une grande prouesse: «Et si avoit encore autres teches, car qui son consel li deist, ja par lui ne fust descovers». Quand il était l'amant d'une dame, il a fait preuve de «mervellouse proece et avoit eu los et pris de sa chevalerie en mainte terre ${ }^{36} »$. L'amour est le moteur de la prouesse: aussi ne faut-il pas s'étonner si, en renonçant à l'amour, Claudas abandonne du même coup les qualités requises pour être un bon chevalier.

La partie centrale de la description juxtapose les deux aspects de Claudas : «ses teces estoient et boines et mauvaises ${ }^{37}$ » (nous soulignons). La syntaxe ne propose pas de synthèse, la répétition de «et» souligne la dualité et le caractère contradictoire de Claudas. Lors de la première mention du personnage, ses qualités étaient d'ailleurs contrebalancées par un «mais» annonçant sa traîtrise. Dans la partie centrale du portrait que dresse le narrateur, la même structure binaire est à l'œuvre, avec un «volentiers» dans le premier membre de la phrase, puis une négation qui annule en quelque sorte le bien-fondé de l'activité qu'il pratique «volontiers » ${ }^{38}$ :

Volentiers aloit au moustier, mais ne faisoit mie granment de bien a povre gent. Moult volentiers levoit matin et manjot, $n e$ ja ne juast as eschés ne as tables ne a autres jeus se moult petit non. En bois aloit volentiers. II. jours ou. III. tout de route, non pas acostumeement. Ses couvenans ne faisoit mie volentiers, mais sovent metoit sus ocoison de barat et de dechevanche (nous soulignons).

Claudas va à l'église, mais n'est pas charitable; il se lève tôt le matin, mais ne goûte guère les distractions d'un homme «courtois»; il chasse, mais seulement de temps en temps. La dernière phrase n'obéit pas exactement

\footnotetext{
35. Lancelot, t. VII, p. 54

36. Lancelot, t. VII, p. 55

37. Lancelot, t. VII, p. 54 .

38. Lancelot, t. VII, p. 54
}

à la même logique. Le début de la phrase, déjà, comporte une négation et montre son caractère félon qui n'est que renforcé dans la suite. Non seulement il a du mal à tenir sa parole, mais aussi, pour y échapper, il peut se montrer trompeur. Cette dernière réflexion oriente habilement la suite de la description, dans laquelle le caractère mauvais et hypocrite de Claudas est mis en avant. Malgré les qualités «et boines et mauvaises», ce sont ces dernières qui dominent.

Analyser les deux portraits en regard l'un de l'autre nous a permis de distinguer la différence de traitement des deux personnages. Le narrateu multiplie les effets pour signaler la dualité de Claudas : il est laid de visage, mais beau de corps; la syntaxe binaire qui intervient à plusieurs reprises pour décrire son caractère et ses occupations reproduit l'ambivalence du personnage. Lancelot, lui, est globalement beau et bon, mais si on analyse plus précisément sa description, nous constatons qu'il combine la sagesse de l'adulte aux caractéristiques de l'enfance. L'entremêlement d'élément divers est au cœur de ce personnage et s'illustre par celui des couleurs de son teint. C'est le résultat d'une nuance de trois couleurs qui s'accorden (blanc, brun, rouge). La troisième est fondamentale. Chez Lancelot, il n'y a ni simple juxtaposition, comme c'est le cas pour les couleurs rousse et noire de la chevelure de Claudas, ni confusion des contraires, mais fusion par un juste équilibre. Les frontières entre ses différentes caractéristiques ne se brouillent pas, bien que l'entremêlement aboutit à une intégration, à une synthèse des contraires par la médiation d'un troisième élément. Pourtant, un élément du corps de Lancelot rompt à première vue l'harmonie de ses composantes. Mais cela ne veut surtout pas dire qu'il faille supprimer ce qui est disproportionné, soit son cœur et sa poitrine: ils sont en concorde l'un avec l'autre et marquent son caractère d'exception. C'est parce que Lancelot est le chevalier et l'amant de la reine qu'il se dépasse et se livre à de si grandes prouesses.

Ludivine JAQUIERY - Université de Genève, Département de langues et litteratures françaises et latines médiévales

Esthétique de l'entremêlement dans les portraits du jeune Lancelot et de Claudas de la Terre Déserte dans le Lancelot en prose

Les modèles préconisés par les arts poétiques pour la description des personnes laissent à ceux qui se livrent à l'exercice du portrait la possibilité de renouveler le modèle théorique. Cette étude propose une analyse des de renouveler le modele thoriqu. Cette étude propose une analyse des portraits, en regard l'un de l'autre, du jeune Lancelot et de Claudas de la Terre Deserte tels quilis apparaissent dans le Lancelot en prose. Le redacteur de ce roman développe les portraits autour des notions d' "entremêlement» des composantes des deux personnages et de "mesure» dans leurs proportions. Si la mesure est dordinaire source dharmonie, le traitement reservé à ces deux personnages prouve plutôt le contraire. Quant à l'«entremêlement", il 
présente un résultat différent selon que l'on considère Claudas ou Lancelot. Le premier reste ambivalent, double, tandis que le second dépasse les éléments contradictoires qui le composent en les intégrant: ces derniers fusionnent en se valorisant mutuellement.

arts poétiques - Claudas de la Terre Déserte - entremêlement - Lancelot Lancelot en prose - mesure - portrait

The Aesthetic of «Entanglement» in the Portraits of the Young Lancelot and of Claudas de la Terre Déserte in the Prose Lancelot

The poetic arts have recommended models for describing characters. However, these models are not fixed and allow anyone who wishes to portray a character to renew them. This study offers a comparative portrait analysis of the young Lancelot and Claudas de la Terre Déserte as they appear in Lancelot en prose. The author of this novel develops the portraits through notions of entremêlement [entanglement] of the components of the two characters and of mesure [measure] of their proportions. If measure is usually a source of harmony, the manner of describing the two characters proves rather the opposite. The results differ for Claudas or Lancelot when entremêlement [entanglement] is considered. The former is ambivalent, double, whilst the latter transcends the contradictory elements that compose him by integrating them. Their merger increases the value of each another.

Poetics Arts - Claudas de la Terre Déserte - entanglement - Lancelot Lancelot en prose - measure - portrait 